Alma Mater Studiorum - Università di Bologna DEPARTMENT OF ECONOMICS

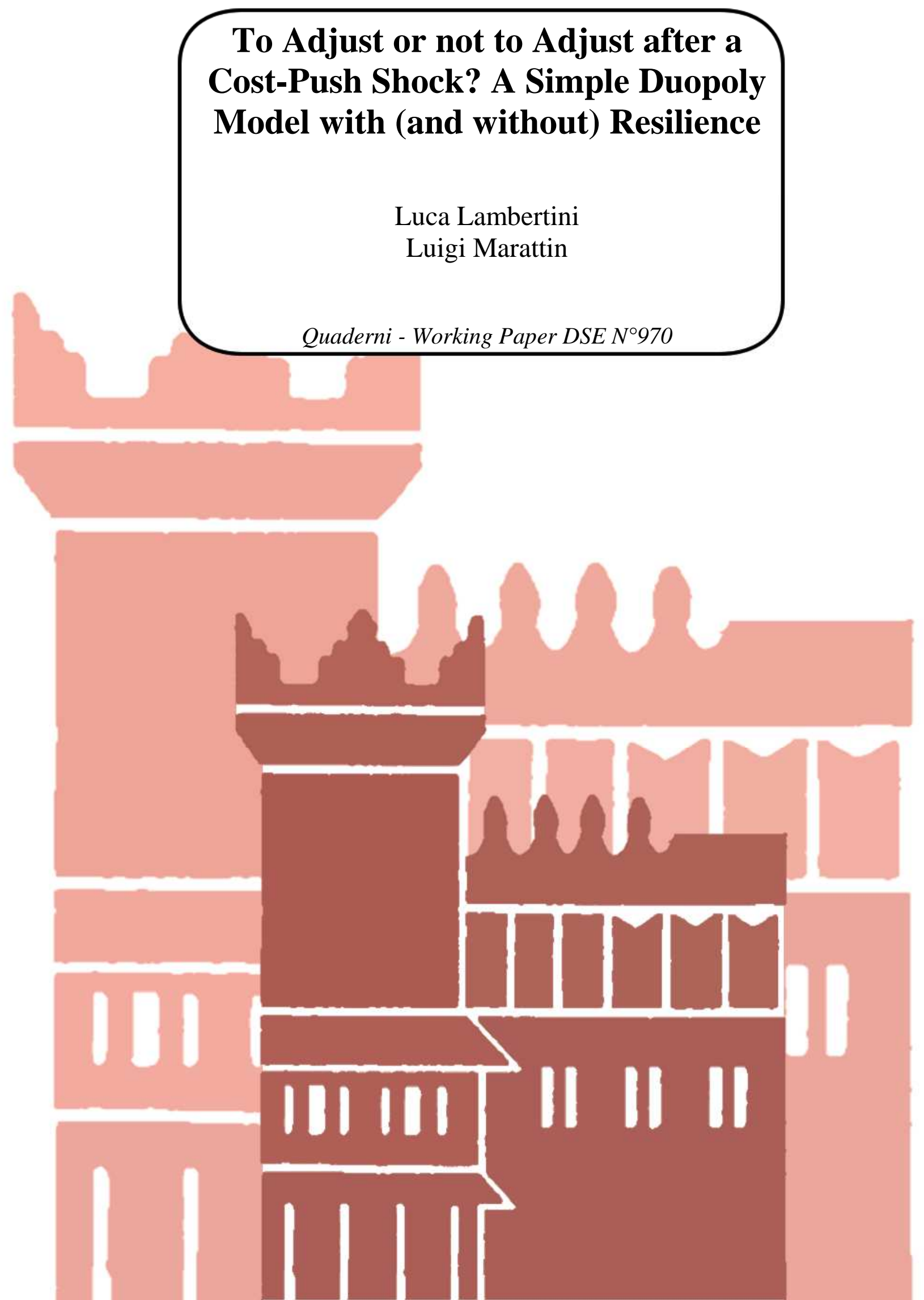




\title{
To Adjust or not to Adjust after a Cost-Push Shock? A Simple Duopoly Model with (and without) Resilience*
}

\author{
Luca Lambertini $^{\dagger} \quad$ Luigi Marattin ${ }^{\ddagger}$
}

October 20, 2014

\begin{abstract}
We characterize the equilibrium in a homogeneous good Cournot duopoly in which firms have the choice to react to a cost-push shock by paying a lump-sum adjustment cost in order to offset the initial rise in marginal cost. Our results show that the size of the shock and the size of the adjustment cost jointly determine the nature and the number of the equilibria generated in the game. In particular, if the adjustment cost is high enough, at least one firm decides not to adjust at the pure strategy equilibrium, and such a partial adjustment by the industry can be socially efficient as well. Some implications of this partial equilibrium analysis about an industry' resilience are outlined.
\end{abstract}

JEL Codes: D43, E30, L13

Keywords: Cournot duopoly, cost-push shock, resilience

*We would like to thank Roberto Cellini for useful suggestions. The usual disclaimer applies

$\dagger$ Corresponding author: Department of Economics, University of Bologna. Strada Maggiore 45, 40126 Bologna, Italy. Email: luca.lambertini@unibo.it; fax: +39-051-2092664; phone: +39-051-2092623.

${ }_{\ddagger}^{\ddagger}$ Department of Economics, University of Bologna. Strada Maggiore 45, 40126 Bologna, Italy. Email: luigi.marattin@unibo.it 


\section{Introduction}

How do oligopolistic firms react to an exogenous cost-push shock when they have a chance to undertake a costly action to offset it? The question is relevant as firms challenged by a loss in competitiveness induced by an increase in production costs are increasingly induced to restore the pre-shock level of profitability by acting on other parts of the cost structure, rather than revising their pricing policy. Actions of this nature are seldom costless.

We propose an analysis of this issue in a simple Cournot duopoly model affected by a shock increasing the level of marginal cost by the same amount for both firms, each of which has to evaluate the profitability of spending a fixed amount of resources to bring back its marginal cost to the initial level, in a fully noncooperative game. Intuitively, the equilibrium outcome depends on the level of the adjustment cost, and the firms' incentives to undertake this costly venture fade away as it increases. In particular, there exists an intermediate range of values of the adjustment cost in which two asymmetric equilibria obtain in pure strategies, such that one firm adjusts while the other doesn't. Interestingly enough, such asymmetry may indeed turn out to be aligned with social preferences, so that a government would raise no objection to an equilibrium outcome characterised by asymmetric levels of productive efficiency and investments.

After carrying out the analysis of the duopoly model, we propose different interpretations of the source and nature of the cost-push shock as well as the reaction to it, linking the ensuing partial equilibrium analysis typically belonging to the theory of industrial organization to far-reaching discussions about the influence of labour unions on firms' innovation incentives (Ulph and Ulph, 1998), the neoclassical adjustment mechanism to the long-run equilibrium after a permanent demand shock (Woodford, 2003) and resilience (Lee et al., 2013). 


\section{The model}

The economy is composed by two identical firms (indexed by $i=1,2$ ) producing a homogeneous good. Consider a time horizon consisting of three periods, $t-1, t$ and $t+1$. At time $t-1$, demand and cost functions are respectively $p=a-Q=a-q_{1}-q_{2}$ and $C_{i}=\underline{c} q_{i}$, where $p$ denotes the price of the product and $q_{i}$ is firm $i$ 's quantity. Marginal cost is given by parameter $\underline{c} \in(0, a)$. Firms play à la Cournot-Nash, and therefore individual profits at time $t-1$ read $\pi(t-1)=(a-\underline{c})^{2} / 9$. Then, between $t-1$ and $t$ (say, overnight), the industry is hit by an exogenous shock shifting the marginal cost $^{1}$ up to $\bar{c} \in(\underline{c}, a)$ and individual profits down to $\pi(t)=(a-\bar{c})^{2} / 9<\pi(t-1)$. Now assume the existence of a symmetric lump-sum adjustment cost $k>0$ that, if paid by the firm, is going to restore the initial level of the marginal cost and therefore also profits (net of $k$ ) at time $t+1$. In other words, firms may implement a costly action so as to offset the initial increase in marginal cost from $\underline{c}$ to $\bar{c}$. There are many ways we can rationalize such a cost: R\&D activities for a process innovation measured by $\bar{c}-\underline{c}$, or a bargaining process aimed at reducing input costs (labor, capital or raw materials), assuming they are exchanged in non-competitive markets. For our purpose, all that matters is that such a reaction to the shock is costly. If both firms decide to implement the reaction after the marginal cost shock has occurred, then at $t+1$ individual profits are given by the following expression:

$$
\pi^{A A}=\frac{(a-\underline{c})^{2}}{9}-k=\pi(t-1)-k
$$

where superscript $A A$ mnemonics for symmetric adjustment. Profits $\pi^{A A}$ are positive for all

$$
k \in\left(0, k^{A A}\right), k^{A A}=\frac{(a-\bar{c})^{2}}{9}
$$

\footnotetext{
${ }^{1}$ For the sake of simplicity, we are focussing on a shock affecting marginal cost, but the ensuing analysis would go through unmodified if we instead assumed the shock to decrease demand. That is, a simple reformulation of market size $a-c=\sigma$ would allow us to model a shock driving market size down from $\bar{\sigma}$ to $\underline{\sigma}$, with entirely analogous results.
} 
For any $k>k^{A A}$, the size of the shock is large enough to eliminate any incentive for firms to carry out the symmetric adjustment.

If both firms decide instead not to adjust, then profits remain at the level identified by $\pi(t)$, and can be usefully relabelled as

$$
\pi^{N N}=\frac{(a-\bar{c})^{2}}{9}
$$

where superscript $N N$ indicates that neither firm is adjusting.

In case of asymmetric adjustment, we identify with superscript $A$ the firm which, after the cost-push shock, decides to pay $k$ to bring its marginal cost back to the initial level $\underline{c}$, and with superscript $N A$ the firm which does not. In order for the industry to remain a duopoly with both firms selling positive output levels, we impose the condition $\bar{c} \in(\underline{c},(a+\underline{c}) / 2)$. Borrowing from the jargon typical of the literature on the economics of innovation, this assumption entails that the shock is minor, or non-drastic. ${ }^{2}$ Asymmetric equilibrium profits are the following:

$$
\begin{aligned}
\pi^{A N} & =\frac{(a-2 \underline{c}+\bar{c})^{2}}{9}-k \\
\pi^{N A} & =\frac{(a-2 \bar{c}+\underline{c})^{2}}{9}
\end{aligned}
$$

For all $\bar{c} \in(\underline{c},(a+\underline{c}) / 2),(5)$ is strictly positive. The positivity of $(4)$ is ensured for all

$$
k \in\left(0, k^{A N}\right), k^{A N}=\frac{(a-2 \underline{c}+\bar{c})^{2}}{9}>0 \text { as } \underline{c}<\bar{c}
$$

This condition provides an upper bound on the size of the adjustment cost in the asymmetric case; if it is violated, no firm will unilaterally undertake the adjustment.

Having now fully characterised the spectrum of market subgames and their outcomes, we may design a reduced-form representation of the game

\footnotetext{
${ }^{2}$ See, for instance, the debate on the persistence of monopoly (Gilbert and Newbery, 1982; Reinganum, 1983), where the same asymmetric Cournot duopoly is used to assess whether an incumbent may invest more than a potential entrant in order to keep monopoly power. For more, see Tirole (1988).
} 
following the rise in marginal cost, where each firm has to decide whether to adjust or not to the cost-push shock, i.e., whether to pay $k$ and bring back marginal costs to $\underline{c}$. Such a reduced form is represented by Matrix 1 defined in the binary space of discrete strategies $(A, N)$, and defines a noncooperative game with complete, symmetric and imperfect information (i.e., firms move simultaneously).

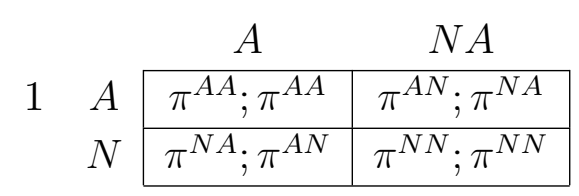

Matrix 1

The equilibrium outcome(s) and Pareto-efficiency (whenever the latter property is indeed relevant) are determined by the signs of the following expressions:

$$
\begin{aligned}
& \pi^{A N}-\pi^{N N}=\frac{(a-2 \underline{c}+\bar{c})^{2}}{9}-k-\frac{(a-\bar{c})^{2}}{9} \\
& \pi^{A A}-\pi^{N A}=\frac{(a-\underline{c})^{2}}{9}-k-\frac{(a-2 \bar{c}+\underline{c})^{2}}{9} \\
& \pi^{A A}-\pi^{N N}=\frac{(a-\underline{c})^{2}}{9}-k-\frac{(a-\bar{c})^{2}}{9}
\end{aligned}
$$

Expression (7) measures the incentive for a firm to pay the adjustment cost $k$ when the rival does not react to the cost-push shock. If it is positive then the firm unilaterally adjusts, otherwise it doesn't. Expression (8) tells us whether for either firm it is convenient to reduce marginal cost to its preshock level when also the rival does, and this happens only if the r.h.s. is positive. Finally, expression (9) allows to Pareto-rank the symmetric payoffs appearing along the main diagonal of Matrix 1. If it is positive, the symmetric adjustment is always Pareto-superior to the symmetric lack thereof.

It can be easily checked that $(7)$ is positive for all $k \in(0, \bar{k})$, where:

$$
\bar{k}=\frac{4(a-\underline{c})(\bar{c}-\underline{c})}{9}
$$


Expression (8) is positive for all $k \in(0, \widetilde{k})$, where:

$$
\widetilde{k}=\frac{4(a-\bar{c})(\bar{c}-\underline{c})}{9}
$$

Finally, (9) is positive for all $k \in(0, \widehat{k})$, where:

$$
\widehat{k}=\frac{(2 a-\underline{c}-\bar{c})(\bar{c}-\underline{c})}{9}
$$

The characterization of the equilibrium of the game is therefore given by the size of the adjustment cost $k$ with respect to the three above thresholds. According to that, in fact, expressions (7)-(9) assume different signs and consequently give rise to different strategic interaction's outcome. Before analysing that issue, it is necessary to order $k_{1}, k_{2}$ and $k_{3}$, as we do in the following lemma.

Lemma $1 \bar{k}>\widetilde{k}>\widehat{k}$ for any $a>\bar{c}>\underline{c}$.

Proof. Since $\bar{c}>\underline{c}, \bar{k}$ is obviously higher than $\widetilde{k}$. Also, we know that $\widetilde{k}-\widehat{k}=(2 a+\underline{c}-3 \bar{c})(\bar{c}-\underline{c}) / 9>0$ as $\bar{c}>\underline{c}$ and, the cost differential being non-drastic, $\bar{c} \in(\underline{c},(a+\underline{c}) / 2)$ to ensure the non-negativity of outputs in the asymmetric cases. Finally, $\bar{k}>\widehat{k}$ is ensured by the fact that $\bar{k}-\widehat{k}=$ $(2 a-3 \underline{c}+\bar{c})(\bar{c}-\underline{c}) / 9>0$ because $a>\bar{c}>\underline{c}$.

Additionally, it is obvious that $k^{A N}>k^{A A}>\widetilde{k}>\widehat{k}$ for all $a>\bar{c}>\underline{c}$, with

$$
\lim _{\bar{c} \rightarrow \frac{a+\underline{c}}{2}} k^{A A}=\widetilde{k}
$$

while comparing $k^{A A}$ and $\bar{k}$ one finds the following:

Lemma $2 k^{A A}>\bar{k}$ for all $\bar{c} \in(\underline{c},(a+3 \underline{c}) / 4)$. The opposite holds for all $\bar{c} \in((a+3 \underline{c}) / 4, a)$.

Having established that, we are ready to characterize the equilibrium according to the size of the adjustment cost. This is done in Figure 1, drawing $\left\{\bar{k}, \widetilde{k}, \widehat{k}, k^{A A}, k^{A N}\right\}$ in the space $(\bar{c}, k)$. Taking into account constraints (2) and (6), the admissible parameter range is identified by $k \in\left(0, k^{A A}\right)$. This allows us to identify four different areas: 
- Area I, in which $k \in(0, \widehat{k})$. Here, $\pi^{A A}>\pi^{N A}, \pi^{A N}>\pi^{N N}$ and $\pi^{A A}>\pi^{N N}$.

- Area II, in which $k \in(\widehat{k}, \widetilde{k})$. Here, $\pi^{A A}>\pi^{N A}, \pi^{A N}>\pi^{N N}$ and $\pi^{A A}<\pi^{N N}$.

- Area III, in which $k \in\left(\widetilde{k}, \min \left\{\bar{k}, k^{A A}\right\}\right)$. Here, $\pi^{A A}<\pi^{N A}$ and $\pi^{A N}>$ $\pi^{N N}$.

- Area IV, in which $k \in\left(\bar{k}, k^{A A}\right)$ and $\bar{c} \in((a+3 \underline{c}) / 4, a)$. Here, $\pi^{A A}<$ $\pi^{N A}, \pi^{A N}<\pi^{N N}$ and $\pi^{A A}<\pi^{N N}$.

Figure 1 Equilibrium analysis in the space $(\bar{c}, k)$.

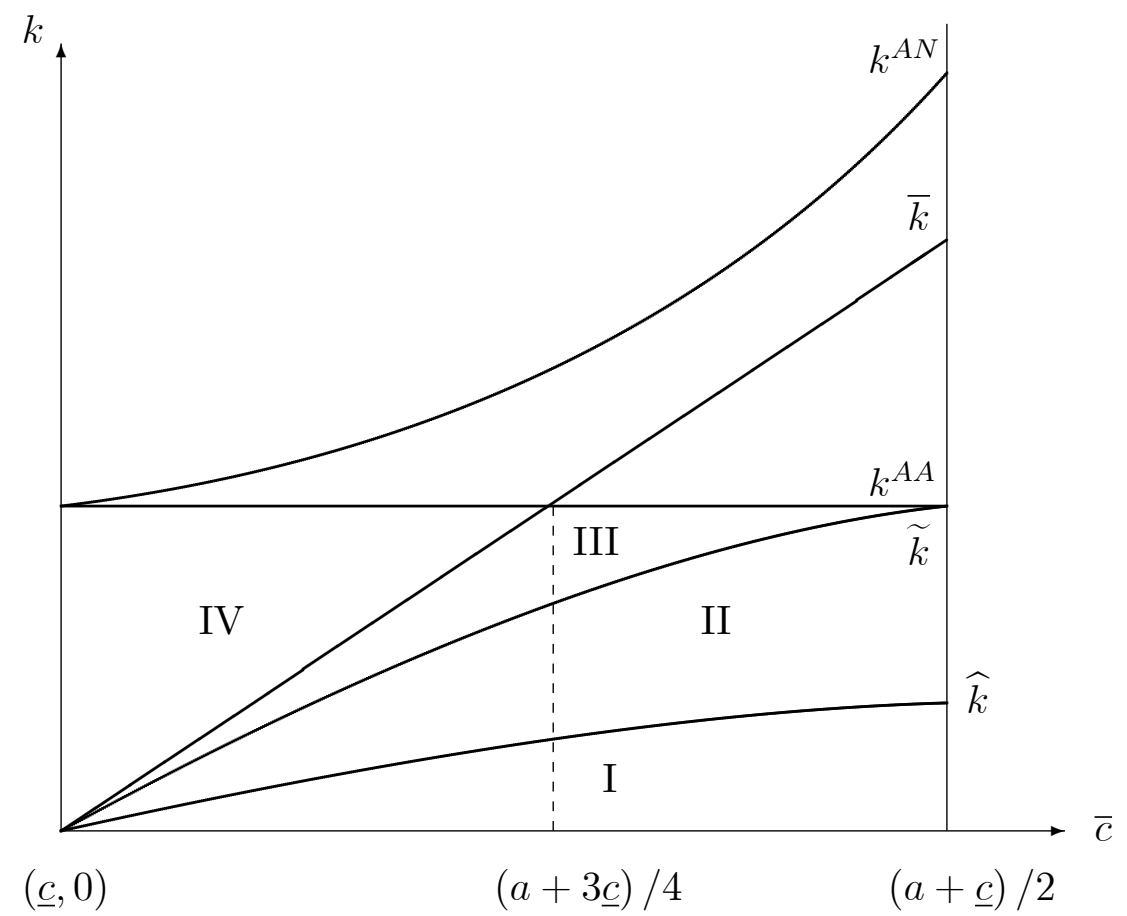

On these basis, we may formulate: 
Proposition 3 Depending on the level of the adjustment cost $k$, the equilibrium outcome of the reduced-form game presented in Matrix 1 is the following:

- In area $I,(A, A)$ is the unique pure-strategy equilibrium at the intersection of dominant strategies, and it is Pareto-efficient for firms.

- In area II, $(A, A)$ is again the unique pure-strategy equilibrium at the intersection of dominant strategies, but it is Pareto-efficient as Matrix 1 portrays a prisoners' dilemma.

- In area III, Matrix 1 is a chicken game with two pure-strategy equilibria along the secondary diagonal, $(A, N A)$ and $(N A, A)$.

- In area $I V,(N A, N A)$ is the unique pure-strategy equilibrium at the intersection of dominant strategies, and it is Pareto-efficient for firms.

The intuitive interpretation of Proposition 3 is straightforward: as the reaction to the cost-push shock becomes more costly, firms obviously are less inclined to undertake the investment required to bring marginal cost back to its initial level, and this makes it harder for the industry to symmetrically adjust as $k$ increases. In the range $k \in\left(\widetilde{k}, \min \left\{\bar{k}, k^{A A}\right\}\right)$, the industry may do so taking into consideration the equilibrium in mixed strategies, which becomes relevant in presence of the two pure-strategy equilibria generated by the chicken game. Having dealt with firms' profit incentives, we have to assess the social welfare consequences of adjustment (or the lack thereof), in order to ascertain (i) whether it is socially desirable, and, if so, (ii) whether profit and social incentives are reciprocally aligned or not.

\section{Welfare appraisal}

Social welfare is defined as the sum of industry profits and consumer surplus. In each of the three relevant cases under consideration, the social welfare level is

$$
S W^{A A}=2 \pi^{A A}+C S^{A A}=\frac{4(a-\underline{c})^{2}}{9}-2 k
$$




$$
\begin{gathered}
S W^{N N}=2 \pi^{N N}+C S^{N N}=\frac{4(a-\bar{c})^{2}}{9} \\
S W^{N A}=S W^{A N}=\pi^{A N}+\pi^{N A}+C S^{N A} \\
=\frac{(a-2 \underline{c}+\bar{c})^{2}}{9}+\frac{(a-2 \bar{c}+\underline{c})^{2}}{9}-k+\frac{(2 a-\bar{c}-\underline{c})^{2}}{9}
\end{gathered}
$$

It is worth noting that the above expressions are strictly positive for all $k \in\left(0, k^{A A}\right)$ and all $\bar{c} \in(\underline{c},(a+3 \underline{c}) / 4)$ since in this region profits are strictly positive. From expressions (14-16), we obtain the following:

$$
\begin{gathered}
S W^{A A}>S W^{N A} \forall k \in\left(0, k_{\alpha}^{S W}\right), k_{\alpha}^{S W}=\frac{(\bar{c}-\underline{c})(8 a-11 \bar{c}+3 \underline{c})}{18} \\
S W^{N A}>S W^{N N} \forall k \in\left(0, k_{\beta}^{S W}\right), k_{\beta}^{S W}=\frac{(\bar{c}-\underline{c})(8 a+3 \bar{c}-11 \underline{c})}{18} \\
S W^{A A}>S W^{N N} \forall k \in\left(0, k_{\gamma}^{S W}\right), k_{\gamma}^{S W}=\frac{2(\bar{c}-\underline{c})(2 a-\bar{c}-\underline{c})}{9}
\end{gathered}
$$

and it is quickly established that $k_{\beta}^{S W}>k_{\gamma}^{S W}>k_{\alpha}^{S W}$ for all $\bar{c} \in(\underline{c},(a+3 \underline{c}) / 4)$. This exercise gives rise to the picture represented in Figure 2, with four relevant areas:

- Area $\mathbb{A}$, in which $k \in\left(0, k_{\alpha}^{S W}\right)$. Here, $S W^{A A}>S W^{N A}>S W^{N N}$.

- Area $\mathbb{B}$, in which $k \in\left(k_{\alpha}^{S W}, k_{\gamma}^{S W}\right)$. Here, $S W^{N A}>S W^{A A}>S W^{N N}$.

- Area $\mathbb{C}$, in which $k \in\left(k_{\gamma}^{S W}, k_{\beta}^{S W}\right)$. Here, $S W^{N A}>S W^{N N}>S W^{A A}$.

- Area $\mathbb{D}$, in which $k \in\left(k_{\beta}^{S W}, k^{A A}\right)$. Here, $S W^{N N}>S W^{N A}>S W^{A A}$.

Accordingly, we may formulate:

Proposition 4 Depending on the level of the adjustment cost $k$, social preferences can be summarised as follows:

- In area $\mathbb{A}$, symmetric adjustment by the entire industry is socially efficient. 
- In areas $\mathbb{B}$ and $\mathbb{C}$, asymmetric adjustment by a single firm is socially efficient.

- In area $\mathbb{D}$, it is socially efficient that neither firm adjusts.

Figure 2 Welfare analysis in the space $(\bar{c}, k)$.

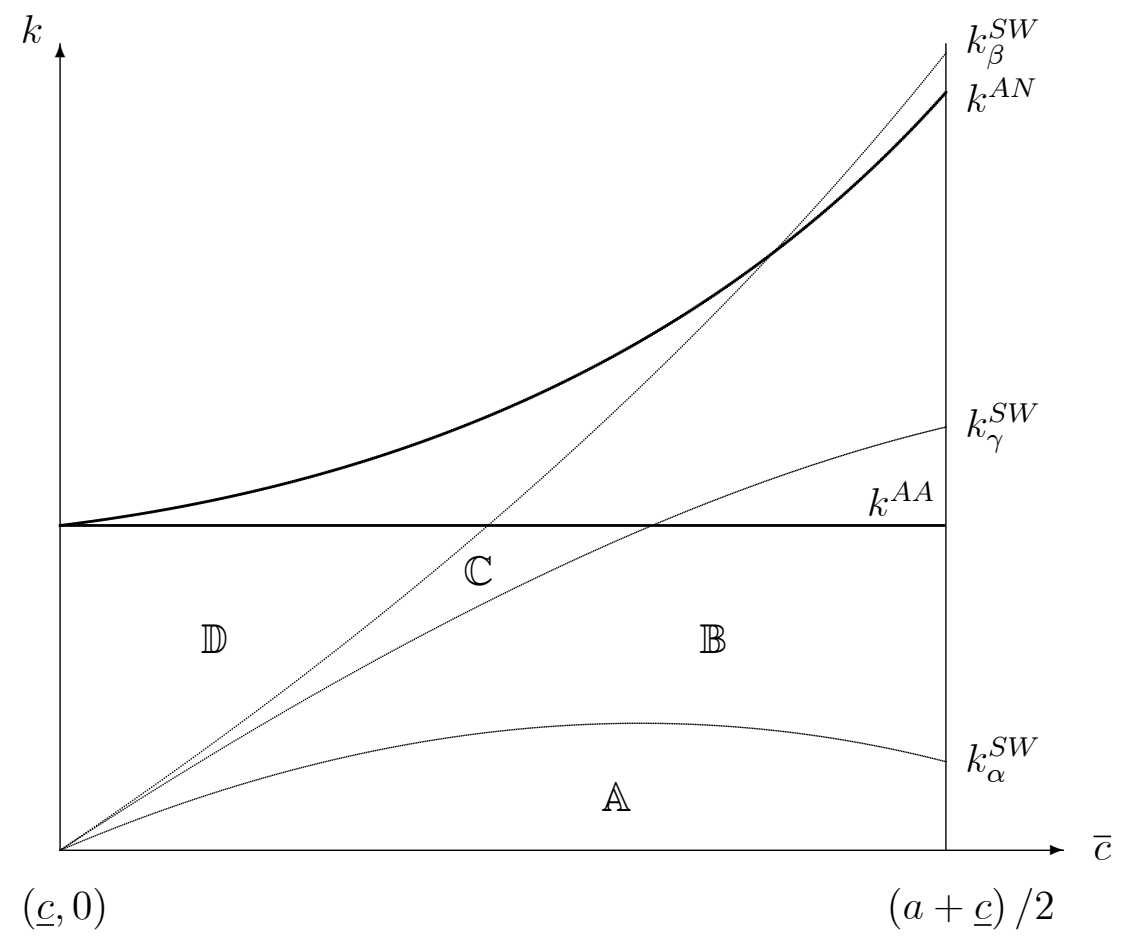

As for firms' incentives, also here we see that - not surprisingly - symmetric adjustment is efficient provided that $k$ is sufficiently low. The last step consists in tackling the issue of the alignment between private and social incentives. This task can be performed putting together all of the critical threshold of $k$ in a single graph. This is done in Figure 3, revealing that there exist three areas wherein alignment does emerge: 
- In area $(*)$, identified by $k \in\left(0, \min \left\{\widehat{k}, k_{\alpha}^{S W}\right\}\right)$, the unique equilibrium $(A, A)$ involving adjustment by the entire industry is Paretoefficient for firms as well as from a social standpoint.

- In area $(* *)$, asymmetric adjustment by a single firm is the outcome of the chicken game, and is also socially optimal.

- In area $(* * *),(N, N)$, i.e., no adjustment by either firm, is the unique equilibrium generated by firms' strategic interplay and is also socially efficient.

Figure 3 Private and social incentives in the space $(\bar{c}, k)$.

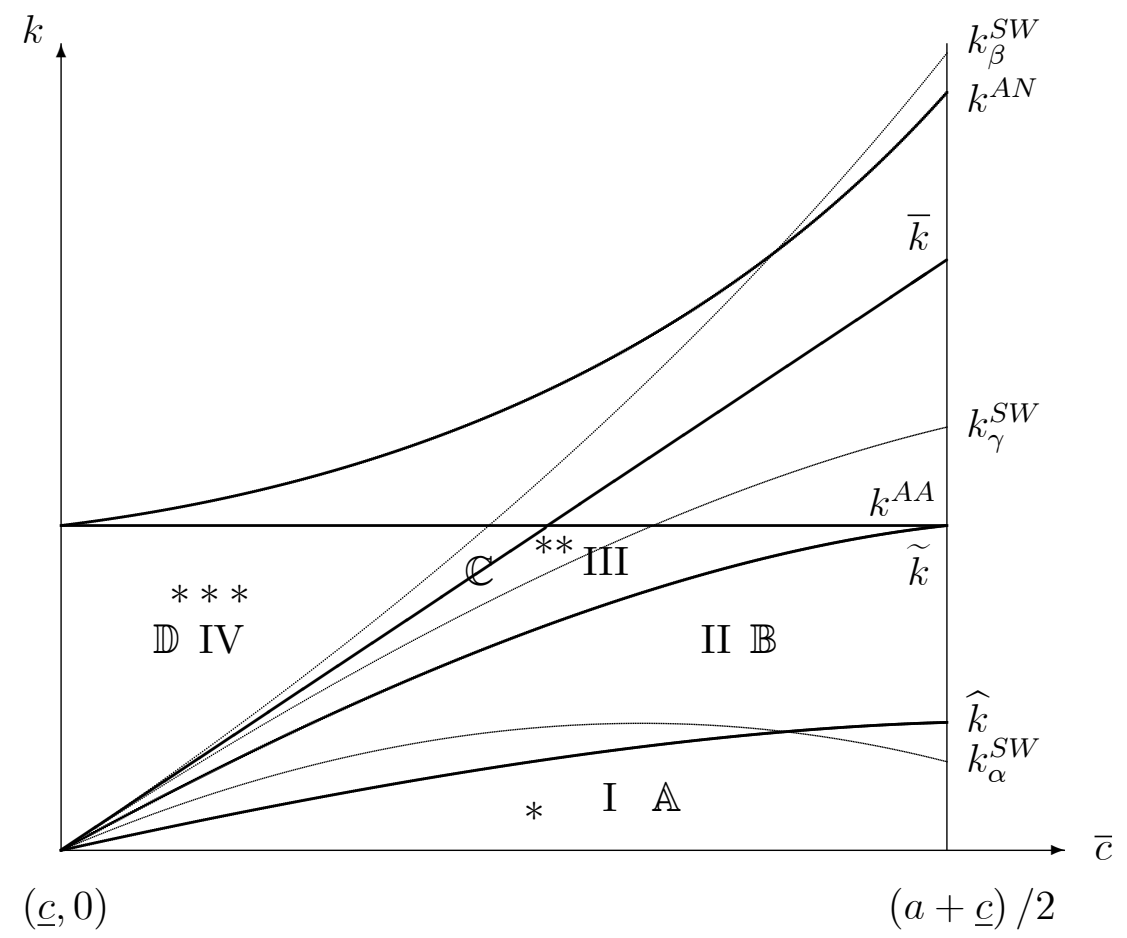




\section{Implications and concluding remarks}

In the foregoing exposition, we did not elaborate on the source of the shock affecting firms' marginal cost. This can be generated by fiscal policy actions (such as increase in tax rates on production or inputs) or institutional features (labor unions'demands causing an increase in real wage). Or, additionally, it may be the outcome of an increase in the cost (or scarcity) of raw materials or fossil fuels, with analogous consequences. Adopting a wider perspective ranging far outside the boundaries of partial equilibrium analysis, the role played by cost-push shocks has indeed received a great deal of attention in the macroeconomic literature (see Ravenna and Walsh, 2006; Chowdhury et al., 2006; Henzerl, 2009). If these motives lie behind the marginal cost increase, then the adjustment cost required to bring it back to its initial level can be interpreted as either an R\&D investment or the cost implied by the bargaining process between firms and labor unions to reach an agreement on the reconstruction of the status quo ante. ${ }^{3}$

Furthermore, the simple model laid out in this note might paves the way for an introductory theoretical treatment of the resilience issue that is, having been borrowed from physics, is currently attracting an increasing amount of attention in economics. Adopting the interpretation according to which resilience is a system's capacity to bounce back from a shock to its previous state (cf. Martin and Sunley, 2014), our analysis shows that (i) resilience is not necessarily to be expected to emerge because firms may not be willing to carry out the required costly efforts, and more importantly (ii) that resilience may neither be socially desirable, for the very same reason.

\footnotetext{
${ }^{3}$ Alternatively, the objectives and actions of labor unions may affect firms' innovation incentives. See Ulph and Ulph (1998), Calabuig and Gonzalez-Maestre (2002); Haucap and Wey (2004); Mukherjee and Pennings (2011), inter alia.
} 


\section{References}

[1] Calabuig, V. and M. Gonzalez-Maestre (2002), Union Structure and Incentives for Innovation, European Journal of Political Economy 18, $177-92$.

[2] Chowdhury, I., M. Hoffman and A. Schabert (2006), Inflation Dynamics and the Cost Channel of Monetary Transmission, European Economic Review 50, 995-1016.

[3] Farber, H. (1986), The Analysis of Union Behavior, in Ashenfelter, O. and R. Layard (eds), Handbook of Labour Economics, Vol. 2, Amsterdam, North-Holland.

[4] Gilbert, R. and D. Newbery (1982), Preemptive Patenting and the Persistence of Monopoly, American Economic Review 72, 514-26.

[5] Haucap, J. and C. Wey (2004), Unionisation Structures and Innovation Incentives, Economic Journal 114 (Conference Papers), C149-65.

[6] Henzel, S., O. Hulsewig, E. Mayer and T. Wollmershauser (2009), The Price Puzzle Revisited: Can the Cost Channel Explain a Rise in Inflation After a Monetary Policy Shock?, Journal of Macroeconomics 31, 268-89.

[7] Lee, A.V., J. Vargo and E. Seville (2013), Developing a Tool to Measure and Compare Organisations' Resilience. Natural Hazards Review 14, $29-41$.

[8] Mukherjee, A. and E. Pennings (2011), Unionization Structure, Licensing and Innovation, International Journal of Industrial Organization 29, $232-41$.

[9] Ravenna, F., C.E. Walsh (2006), Optimal Monetary Policy with the Cost Channel, Journal of Monetary Economics 53, 199-216.

[10] Reinganum, J. (1983), Uncertain Innovation and the Persistence of Monopoly, American Economic Review 73, 741-48. 
[11] Martin, R. and P. Sunley (2014), On the Notion of Regional Economic Resilience: Conceptualization and Explanation, Journal of Economic Geography, forthcoming.

[12] Tirole, J. (1988), The Theory of Industrial Organization, Cambridge, MA, MIT Press.

[13] Ulph, A. and Ulph, D. (1998), Labour Markets, Bargaining and Innovation, European Economic Review 42, 931-39. 


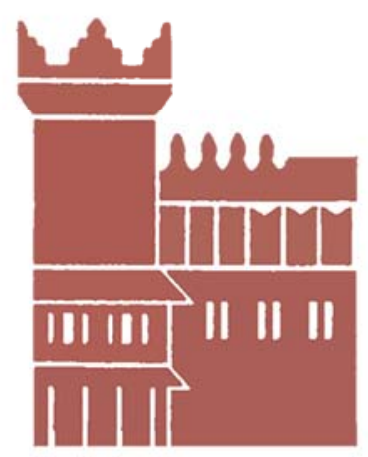

Alma Mater Studiorum - Università di Bologna DEPARTMENT OF ECONOMICS

Strada Maggiore 45

40125 Bologna - Italy

Tel. +39051 2092604

Fax +390512092664

http://www.dse.unibo.it 